Service social

\title{
Les vagabonds du rêve - Vers une société de marginaux, par Marc Lesage, Montréal, Boréal Express, 1986, 119 pages.
}

\section{Yves Hurtubise}

Volume 35, numéro 3, 1986

Les jeunes et le travail social

URI : https://id.erudit.org/iderudit/706325ar

DOI : https://doi.org/10.7202/706325ar

Aller au sommaire du numéro

Éditeur(s)

École de service social de l'Université Laval

ISSN

1708-1734 (numérique)

Découvrir la revue

Citer ce compte rendu

Hurtubise, Y. (1986). Compte rendu de [Les vagabonds du rêve - Vers une société de marginaux, par Marc Lesage, Montréal, Boréal Express, 1986, 119 pages.]

Service social, 35(3), 475-478. https://doi.org/10.7202/706325ar d'utilisation que vous pouvez consulter en ligne.

https://apropos.erudit.org/fr/usagers/politique-dutilisation/ 


\section{RECENSIONS}

Les vagabonds du rêve - Vers une société de marginaux, par Marc LESAGE, Montréal, Boréal Express, 1986, 119 pages.

Marc Lesage est un militant syndical et un permanent de la C.S.N. Sociologue, à l'affût des signes annonciateurs de changement, il nous livre ici une réflexion sur de nouveaux phénomènes sociaux qui devraient préoccuper les organisations syndicales, mais aussi celles et ceux qui travaillent avec ou auprès des marginaux, ou qui réfléchissent à l'avenir de notre société. À la parution du livre, les grands médias écrits ne se sont pas trompés : ils ont perçu un signe de changement et en ont témoigné.

Le changement d'ailleurs est à situer à deux niveaux : celui dont nous parle Lesage, mais aussi celui dont l'écrit de l'auteur témoigne : la remise en question des fondements théoriques qui ont soutenu l'action de certains syndicats et groupes populaires au Québec. Cette réflexion me semble marquer, à ce point de vue, un virage qui était déjà perceptible et qui est maintenant clair.

Dans le cadre d'une thèse de doctorat, l'auteur part à la recherche d'un nouveau prolétariat qu'il a senti naître au temps où il travaillait avec des syndiqués en lutte, et surtout au moment où il appuyait la formation du Syndicat des sans-emploi, à la C.S.N. C'est à une quinzaine de personnes, qui lui étaient plus ou moins liées étroitement, qu'il proposa d'entreprendre une réflexion qui s'est déroulée sur le thème de la précarité de l'emploi et de l'exclusion du marché du travail. Huit participants avaient moins de 30 ans. La démarche proposée, développée par Alain Touraine et son équipe, se voulait une "intervention sociologique"; une annexe au livre donne quelques paramètres de cette approche. L'auteur indique, dans le texte et dans cette annexe, les accrocs qu'il s'est permis à l'égard de cette méthode.

Dans le premier chapitre, on pose le problème de l'hétérogénéité croissante du prolétariat dans une conjoncture occidentale de restructuration de l'économie. De plus en plus de travailleurs - singulièrement les femmes et les jeunes - sont exclus du marché du travail stable et se retrouvent dans des emplois précaires, mal payés, sans mesures de protection sociale, et ne pouvant guère compter sur un syndicat pour négocier leurs conditions de travail. Ce phénomène bouleverse les analyses traditionnelles sur la classe ouvrière ; il interroge non seulement les sociologues mais aussi les centrales syndicales qui, au Québec comme ailleurs en Occident, ont toujours voulu rassembler les travailleurs pour mieux lutter contre le patronat et l'État. Or, nous dit Lesage, force est de constater l'échec des syndicats devant cet éclatement du marché du travail. Comment recréer une conscience ouvrière alors que les caractéristiques socio-économiques des salariés nous offrent un portrait fait de morcellement et de divisions? Il y a là un défi au mouvement syndical, dont des centrales comme la C.G.T. et la C.F.D.T. en 
France, la C.G.I.L. en Italie, et la C.S.N. au Québec, ont pris bonne note. Toutes s'interrogent sur les virages à prendre pour s'ajuster aux nouvelles réalités; la C.S.N., quant à elle, a favorisé la création d'un syndicat des sans-emploi, dont l'expérience sera analysée par le groupe de Lesage.

Dans le deuxième chapitre, l'auteur tente de cerner les caractéristiques de ce nouveau prolétaire, ce qui le distingue de l'ouvrier " traditionnel "; mais, ditil : " [...] la place des nouveaux prolétaires dans les rapports sociaux de production n'est pas facile à déterminer " (p. 36). II ne s'agit pas d'une classe sociale (p. 41) mais d'une catégorie sociale dont la conscience serait différente de la conscience ouvrière traditionnelle, et pour qui le travail et le système de production sont des "points de repères et [des] axes privilégiés de ruptures et de propositions" (p. 41). Cette conscience néo-prolétarienne comprend deux volets: une conscience alternative, qui cherche une autre logique de croissance (plus qualitative, plus sociale) et une conscience scandaleuse, qui refuse la culture industrielle.

Reprenant les concepts de Touraine - identité, opposition, totalité Lesage tente de préciser les contours de cette nouvelle conscience. Son identité comporte plusieurs dimensions : refus du travail aliénant et abrutissant, adhésion au travail de communication, de relations sociales, de création artistique ou intellectuelle ; le néo-prolétaire serait prêt à laisser un travail " bête " pour partir en voyage, retourner aux études, etc. II préfère un travail intéressant temporaire et peu rémunéré à un travail ennuyant. Il est instable, dit l'auteur, mais disponible.

Son opposition n'est pas tant aux patrons et aux gestionnaires de l'organisation qu'aux technocrates, aux créateurs d'illusion, aux « metteurs en scène du sens du travail et de sa finalité ". Le néo-prolétaire n'est pas absent des luttes et ne lutte pas que sur le plan politique. "Il investit les mouvements sociaux, produit des pratiques alternatives, évite l'État partout où il peut le faire et ne s'en remet à lui que pour des questions précises ou lorsqu'il n'a plus d'autres choix [...] il lutte au contraire pour la réappropriation du social à travers la recherche de la maîtrise des lieux et des temps de sa vie quotidienne." (P. 50.)

Le nouveau prolétaire a une conscience qui ne se réduit pas à la sphère du travail. II perçoit de grands enjeux qui impliquent la survie de la planète. Sa critique du travail n'est qu'une critique partielle de ce qu'il perçoit comme enjeu fondamental : pourrons-nous continuer à vivre sur cette planète ? Lesage croit qu'il ne s'agit pas d'un désengagement, d'un retrait, mais plutôt "d'une lucidité certaine de véritables enjeux sociétaux" (p. 51).

Les nouveaux prolétaires se diviseraient en deux catégories, tout comme dans le mouvement ouvrier traditionnel ; la première est formée des "scandaleux", ceux qui refusent l'organisation sociale : le blouson noir, le punk dur, le voleur à domicile, la prostituée, mais aussi l'artiste décroché, le musicien de la rue, le grand voyageur qui fuit en Californie ou ailleurs. La seconde catégorie est formée des néo-prolétaires " alternatifs » qui, tout en étant en rupture, s'engagent dans des projets pour vivre, ici et maintenant, de nouvelles valeurs, de nouveaux modes de relations au travail et à la société. C'est cette catégorie qui interpelle 
davantage le syndicalisme pour que les nouvelles luttes concernent la qualité de vie, pour qu'on se préoccupe du sort de la planète, pour qu'on milite autrement. "C'est [...] la revanche de la conscience libertaire et écologiste sur la conscience laborieuse et industrialiste qui a toujours dominé le mouvement ouvrier." (P. 53.)

Au troisième chapitre, l'auteur présente des extraits d'entrevues significatifs de ce qu'il veut démontrer. J'en retiens une impression d'avoir déjà entendu pareils témoignages, d'en avoir lus dans la littérature dite "alternative" et écologiste. L'élément novateur, si je puis m'exprimer ainsi, provient davantage de la qualité des personnes qui témoignent et de celui qui rapporte leurs propos. Ici, ce sont des gens qui font ou ont fait partie de la mouvance syndicale, qui ne peuvent être soupçonnés de collusion avec les patrons ou d'opérer un repli sur soi. Au contraire, ils participent à de nouvelles valeurs et cherchent de nouvelles voies. Leurs critiques du mode d'organisation sociale incluent une critique du syndicalisme, un appel à de nouvelles sensibilités, à de nouvelles formes de militance.

Au quatrième chapitre, Lesage aborde une question cruciale : comment se fait-il que les exclus et les marginalisés du marché du travail ne réussissent pas à se constituer en mouvement, ni à joindre les rangs des organisations qui prétendent défendre leurs intérêts? Le groupe, dirigé par Lesage, a entrepris l'étude de cette question, par le biais d'une réflexion sur le Syndicat des sansemploi. Ce qui l'intéresse dans ce cas précis, c'est qu'il s'agissait de la formation d'un syndicat, au sens traditionnel du terme, qui s'inscrivait dans la structure de la C.S.N., avec comme objectif de regrouper des sans-emploi. Voilà une nouveauté, car des regroupements de sans-emploi et de marginalisés du marché du travail existent au Québec, mais à l'extérieur des structures syndicales, même s'ils sont souvent en alliance avec les syndicats. L'hypothèse de l'échec du syndicat est construite sur deux niveaux. D'une part, il s'expliquerait par le caractère novateur de cette forme d'action, par l'absence d'un terrain de luttes circonscrit, par la diversité de ses membres et de ses responsables, par l'absence de services particuliers et constants, par des tensions et des conflits internes. Au deuxième niveau, plus fondamental - dit Lesage - , l'absence d'identification des sansemploi à leur situation socio-économique et l'absence d'incitations sélectives expliqueraient l'échec. La reconnaissance de ces deux facteurs me semble stimulante pour la réflexion et l'action des intervenants et militants, car elle bouleverse des idées reçues dans la gauche québécoise.

Lesage nous rapporte que les chômeurs et les marginalisés du marché du travail ne se définissent pas comme tels; ils sont en période transitoire ou dans une position sociale qu'ils ne valorisent pas. Comment, dès lors, construire un mouvement sur une identification qu'on rejette ou joindre les rangs d'une organisation syndicale qui regroupe des travailleurs qui sont tellement différents de nous, voire qui apparaissent comme des privilégiés? La question est de taille, car elle concerne non seulement le type d'organisation à mettre sur pied mais aussi les rapports entre le mouvement ouvrier et celui des marginalisés. L'adéquation entre le mouvement syndical et le mouvement populaire comme parties 
d'un mouvement social plus vaste mérite d'être reconsidérée ; Lesage nous y invite.

L'autre hypothèse concernant l'absence d'incitations individuelles à adhérer et militer au Syndicat des sans-emploi fera grincer bien des militants purs et durs. L'auteur lui-même prend des précautions avant de l'avancer; car la militance a horreur de l'intérêt personnel. Militer, pour certains, c'est le missionnariat, se donner à une cause. Force est de constater une baisse sensible de la militance, même si les raisons de le faire $n$ 'ont pas diminué, loin de là. On invite le lecteur à réfléchir sur les conditions d'adhésion et de militance à un groupe. Plus largement, je dirais que c'est la place des individus dans les causes collectives qui est en jeu.

En conclusion, l'auteur convie à une réflexion approfondie sur un syndicalisme renouvelé qui ferait place - peu importe la structure - aux vagabonds du rêve, de telle sorte qu'ils puissent participer de plein droit à l'élaboration de ses nouveaux fondements. "L'émergence de nouveaux sujets prolétaires exige de nouvelles expertises de la part des organisations qui entendent les représenter et les défendre [...] Par exemple, au moment où l'entreprise, l'État, l'école mettent le philosophe au banc des accusés de l'inutilité sociale et du parasitisme, n'est-il pas grand temps de le revoir, de réfléchir avec lui sur le sens du travail et de la vie? Ne serait-il pas aussi pertinent d'analyser avec le psycho-sociologue les relations de domination au sein même de nos assemblées syndicales? De penser avec l'écologiste les conséquences de telles productions sur notre mieux-être ? " (P. 124.)

Rafraîchissant. Pistes de réflexion pour les organisations syndicales mais aussi pour les professionnels du social, pour les organisateurs communautaires et les travailleurs sociaux qui œuvrent avec des exclus de toutes sortes et des individus marginalisés; tous trouveront dans ce livre des témoignages, des affirmations, des interrogations qui leur seront utiles.

École de service social,

Yves HURTUBiSE

Université Laval.

Jeunesse: des illusions tranquilles, collectif sous la direction de Marc-André DeniGer, Jocelyne GAMACHE et Jean-François RenE, Montréal, VLB Éditeur, 1986.

Un des trop rares livres sérieux sur la jeunesse, cet ouvrage a été préparé, conçu et écrit pendant l'Année internationale de la jeunesse pour contribuer à une meilleure compréhension du " phénomène ». D'ailleurs, il n'y a pas une mais "des" jeunesses. Le portrait est complexe et éclaté et mérite qu'on s'y penche plus sérieusement. Les membres du collectif, qui ont mené le projet de publier ce recueil de textes variés, refusent justement les explications unidimensionnelles, comme ils le précisent en introduction: "Notre démarche de groupe procède d'un désir profond de poursuivre le débat au-delà des limites trop contraignantes 